\title{
Interference and Resource Management Strategy for Handover in Femtocells
}

\section{Madiha $\mathbf{R}^{*}$ and Sana $\mathbf{A}$}

Research Centre for Modeling and Simulation, National University of Sciences and Technology, Islamabad, Pakistan

\begin{abstract}
Interference in femtocells due to neighboring femtocells and macrocells is one of the major issues. Handover should be made to reduce interference, if and only if, when resources are available. Otherwise, it will further degrade network performance. Resource management should be made in an efficient manner that will not cause interference between macrocells and neighboring femtocells. Since distance between macro base station (MBS) and Femto Access Point (FAP) is short, therefore, it is very hard to sustain low handover probability when macro user (MUE) moves from MBS to FAP. We proposed handover algorithm for uplink co-channel interference mitigation that will make decision of handover on the basis of time-to-stay (TTS) and Signal to interference plus noise ratio (SINR) thresholds along with efficient resource management mechanism to reduce number of handovers and also resolve interference problem.
\end{abstract}

Keywords: Interference management; Resource management; Time-to-stay; Fractional frequency reuse; Handover decision; Maximal independent set

\section{Introduction}

$3^{\text {rd }}$ Generation Partnership Project (3GPP) introduced Long-Term Evolution (LTE) technology that aims to provide high data rates, low latency and high spectral efficiency. It also aims to provide improved coverage and throughput. It was expected that $4 \mathrm{G}$ will fulfill the needs of users. However, due to dramatic increase in number of users every year, it failed to fulfill user demands for high data rates, capacity and spectral efficiency. Cellular frequency consumes by network operators are limited from several hundred megahertz to several hundred gigahertz. Due to which, it is quite challenging for them to acquire more. Another challenge is deployment of advanced technology that can fulfill the upcoming demands in near future comes at the cost of high energy consumption. It has been reported that $70 \%$ electricity bills come from cellular base stations [1]

In cellular networks, $2 / 3$ of voice calls and $90 \%$ of data usage occur indoor e.g in homes, offices, airports, hospitals, restaurants etc. Unfortunately, due to indoor path loss, users experience bad indoor coverage. Mobile traffics of the whole mobile world are increasing exponentially due to instant increase of mobile users all over the world [2]. It is expected that it will keep on increasing in magnitude every year [3]. Therefore, we need some solution for improving indoor coverage by installing small cells as suggested by cisco [4]. Although LTE was introduced for fulfilling growing real time network services e.g. VoIP, video streaming etc. However, there are still some gaps left i-e: power consumption, spectral efficiency, roaming issues [5]. Femtocells can be deployed indoor for getting high data rates. Visiting macro users can enter within coverage area of femtocells and can start using their services by performing handover. But if macro user only stay within or near femtocell for relatively less time and its SINR value is greater than specific threshold due to less interference, then there is no need for handover. Because it can keep continuing its services with macro base station without causing any interference but if TTS of MUE is greater than certain threshold, then due interference SINR starts degrading. So we need to define thresholds for SINR and TTS as well as need to work on resource management algorithm to avoid unnecessary handovers. Network planning is very important part because the connection between mobile user and base station is dependent on it. Due to which connection requirements should be fulfilled by taking capacity and data rates in account. In next few years, it is estimated that mobile traffic will increase exponentially, which can be creating huge challenge for current 4G technology to handle. To improve capacity and to offload data and services within the heterogeneous cellular network, we need small cells deployment that could accommodate traffic demands in future [6]. We need a low power base station within the macrocell base station. The main advantage of low power base station such as Pico cell, micro cell and femtocells is that it bring network close to UE and they can enjoy high data rates [7]. femto access point (FAP) or home base station which can extend indoor coverage and can integrate with existing technologies i.e. GSM, LTE, WiMax etc on radio access and backhaul connection can be made by using broadband connection i.e. Optical fiber, DSL etc. [8]. To deal with problem of how many small cells are required and how to resolve interference among these cells, an algorithm was proposed for obtaining best locations for deploying small cell and to increase spectral efficiency [9]. Co-channel interference can cause many technical problems because it's a cross talk between femtocell and macrocell as well as interference between neighboring femtocells using same frequency bands.

\section{Related Work}

Recently due to demand of high data rates for mobile services, it is suggested that cell radius and handover should be reduced. Due to which femtocells, microcells and picocells are emerging technologies. Its purpose is to increase indoor coverage as well as provide high data rates. Femtocells are low-power base stations that are installed on the users's premises to provide cellular service within the home or enterprise environment. Typically Femtocells are connected to the Internet and the cellular operator's network via a digital subscriber line

*Corresponding author: Madiha Rasheed, Research Centre for Modeling and Simulation, National University of Sciences and Technology, Islamabad, Pakistan, Tel: +92-51-111-11-6878; E-mail: mrasheed.msse15@rcms.nust.edu.pk

Received November 19, 2018; Accepted November 21, 2018; Published November 28, 2018

Citation: Madiha R, Sana A (2018) Interference and Resource Management Strategy for Handover in Femtocells. J Telecommun Syst Manage 7: 162. doi: 10.4172/2167-0919.1000170

Copyright: $\odot 2018$ Madiha $R$, et al. This is an open-access article distributed under the terms of the Creative Commons Attribution License, which permits unrestricted use, distribution, and reproduction in any medium, provided the original author and source are credited. 
(DSL) router or cable modem, which is why the users' QoS guarantee is still an open issue. Femtocells offer benefits for both subscribers and operators. Subscribers experience better voice service coverage and higher data throughput. Handoff management is necessary due to frequent movement of macro users to femtocells or vice-versa due to short distance. A handover algorithm is proposed earlier [10] to avoid unnecessary handover from macrocells to femtocells in case of temporary femtocells visitors by using mobility pattern scheme. To overcome with problems of call dropping and handover failures, an optimization algorithm was proposed earlier [11] that change handover parameters of hysteresis varying between 0 and $10 \mathrm{~dB}$ as well as time to trigger values specified by $3 \mathrm{GPP}$ by measuring the performance of networks. But main problem with this algorithm was that it was limited for specific scenario and cannot be applied by assuming extreme cases. A handover algorithm was proposed earlier [12] that will make the decision of handover on the basis of RSS, velocity and bandwidth w.r.t hand-in and hand-out. It will measure RSS level and on the basis of which compared the velocity of MS with two predefined values of velocity. If the velocity is less than certain threshold, it will check the bandwidth availability and type of service to take handover decision. This method was effective to reduce cross-tier interference but does not work for co-channel interference. In a study [13], a handover algorithm was proposed by taking estimation of user's state Rms in account, in terms of location as well as velocity of the MUE. Two classes are defined as Real time for voice/video and elastic for data service. According to scheme, if SINR from FBS is greater than that of MBS and Rms is greater than 0 , then handover takes place from femtocells to macrocell. Otherwise, UE will remain attached to its serving MBS. In a study [14], smart handover algorithm is proposed for slow moving UE in which threshold for received signal strength and time to stay is measured for visiting femtocells.

In a study [15], Dutch auction and stochastic approach is used for selecting femtobase station for handover. Dutch auction is used for selecting FBSs that are acting as bidder and MUEs are acting as auctioneer. Whenever, more than one FBS bid simultaneously, it results in collision. Therefore, auction continues until last FBS. While, stochastic election helps in narrow down the bidding area on the basis of resolution and separation distance.

In a study [16], handover decision is made on the basis of data capacity available on the estimated path using probabilistic method. The probabilistic path and residence time in femtocell is measured and further decision for handover is made by comparison of available data capacity on the estimated path. Orthogonal frequency allocation of resources is proposed in a study [17] to avoid co-channel interference between macro cell and femtocells in LTE. Handover request initiated if signal strength from Femto Base station HenB is greater than handover threshold. Handover takes place from macro cell to femtocell only on the basis of available allocated resources to femtocell that are required for continuing services required by MUE. If requested resources are less than that allocated to femtocell, then request is done to resource management server RMS by FBS to allocated resource block (RB). If $\mathrm{RB}$, after expansion, are lower than required, then handover is rejected.

In a study [18], efficient resource management mechanism for reducing interference problem, fractional frequency reuse (FFR) and a heuristic graph-based channel assignment algorithm in femtocell is introduced. By using this method, no channel can be used by both macrocells and femtocells or neighboring femtocells at same time for avoiding interference. FFR is applied by allocating sub-bands to macrocells and femtocells separately to define that MUE and FUE can used from these sub-bands respectively. While, heuristic graph based channel assignment is applied by using maximal independent set (MIS) to find sets of femtocells that can used reuse frequency in a way to avoid co-channel and cross-channel interference.

To reduce interference in two-tier network, resource management similar to cognitive radio was proposed but the resource blocks were controlled statistically. In this case, a table was maintained containing the interferer information among the cells and table is periodically updated. It aims to maximize throughput for femtocells in case of non real-time services while guarantee throughput for macrocell in case of real-time services. There are two types of physical resource block (PRB): primary and secondary. Primary PRB are allocated in a manner that macrocell allocates priority resources to serve its own user and remaining of them is orthogonally allocated among the femtocells. While secondary PRB are allocated to femtocells on demand to meet required throughput. There is a possibility that primary PRBs are already part of any secondary PRBs. So to deal with the problem of inter-cell interference among the neighboring femtocells using same frequent band, the look-up table will interferer and resource blocks are distributed in a way that can minimize the interference because additional PRBs are not orthogonally allocated among macrocell and femtocells [19].

In a study [20], handover strategy for hand-in and hand-out are proposed based on SINR and TTS of MUE/FUE in the macrocell/ femtocells. The handover decision is initiated by comparing TTS of FUE/MUE within the premises of macrocell /femtocells with predefined threshold for TTS. If TTS is greater than threshold, SINR of MBS/FAP is compared with preliminary SINR and actual SINR threshold for making further handover decision. The simulation shows that optimal power when compared with minimal power will give more promising results for increasing SINR and improves network performance in terms of user rate, outage probability OP and network capacity.

In a study [21], handover is made by first determining the velocity of UE moving from macrocell to macro-cell/Femtocells. If it goes below $30 \mathrm{~km} / \mathrm{hr}$, then highest Received signal strength indication (RSSI) of femtocells/macrocell are determined. After that availability of bandwidth and SINR are compared for choosing best option among femtocells/macrocell for handover. The decision for hard handover is taken by taken Received signal strength (RSS) into account. The modification in handover process was also proposed by increasing TTS (time to trigger) window size that shows promising results by reducing handover while decreasing up-link SINR. Moreover, performance of network due to handover, measurement of bandwidth, margin and measurement update period was also taken into account for different parameters. But it doesn't provide any information on delay, signaling overheads and throughput of UE [22].

It has been seen [23], handover in heterogeneous networks on the basis of SINR for vertical handoff (VHO) is more effective then RSS with some limitations to user velocity. The criteria for handover proposed was to calculate the time of stay within the coverage area of WLAN for slow and fast moving vehicles to avoid unnecessary handoff. For which another SINR threshold was taken into account. If condition satisfies, handover will takes place. Otherwise, there will be no handover. The SFR scheme is used for the evenly distribution of resources among the cells but due to change of uneven traffic load among neighboring cells, it can cause problem in performance due to which soft frequency reuse scheme was proposed for semi distribution of resource. In this research, a fractional frequency reuse-based graph connectivity (FFR-GC) mechanism is used which not only reduce traffic load but also avoid interference among teh neighboring cells. Previous 
studies $[18,20]$ are followed for proposing new effective evaluation technique by considering SINR, TTS and resource availability for taking handover decision. Previously, these parameters are not taken in account together for decision but these are very important parameters and they should be evaluated for gaining good network performance.

\section{System Model}

In two-tier femtocell network, small femtocell are installed within the coverage area of macrocell so users within femtocells coverage can connect to FAP to gain high speed and capacity. Battery consumption of femto user will also be minimal as FAP is located nearby. But handover is required when macrocell user become close to the premises of femtocell by taking account different parameters due to the interference. When MUE starts moving towards the femtocell, its distance from macrocell start increasing due to which high power transmssion by MUE to MBS is required. This will result in dead zone area for near-by femtocells, due to which SINR at FAP degrades. FUEs try to overcome interference caused by MUEs by increasing their transmitting power to FAP, will in return caused interference at MBS. An efficient mitigation is required otherwise it will result in the formation of positive interference. For handling this situation, handover is required. But if handover of MUEs is made only on the basis of its location or signal strenght, it would result in unnecessary handover. Due to which, there is need to build some rules to check that either handover is required or not. For that case, two thresholds for SINR are used: one is preliminary SINR threshold which is used for negotiation whenever handover request come while other is actual SINR threshold which is used for emergency handover to avoid degradation or discontinuation of services. Time to Stay (TTS) parameter is used along with SINR to check the predicted time that MUE stays in femtocell premises to decide whether to handover or not. TTS is necessary it will handover only to MUE when it stays longer in femtocell [20]. Another important factor which is mostly ignored in handover cases that either resource is available or not for handover is not mentioned in a study [20]. Most of the time, when handover request comes, handover takes place without checking either assigned frequency in case of handover is used by near-by Femtocell or not which can cause severe interference. Due to which efficient resource allocation sceheme FFR-GC along with MIS algorithm is used to overcome interference [18].

In macrocell, there are $\mathrm{N}$ numbers of nodes randomly distributed in the network. Each macrocell has eNB or MBS which is acting as a base station for macrocell user equipment (MUE) is located outdoor within its coverage while the nodes within the coverage of femtocells are served by femto access point (FAP) or femto HeNB. As shown in the Figure 1, three MUEs are located in the macrocells. These MUEs are connected to MBS in outdoor environment while for indoor environment three femtocells each containing FAP as base station to serve their respective FUE within their coverage. In a Figure 1, FUEs within femtocells are served by their own FAP.

Consider that MUE1 starts moving towards femtocells and away from MBS. As the distance between MUE1 and MBS increases, SINR decrease. When MUE moves into the premises of femtocells especially within the indoor environment, it needs to transmit with high power due to wall's penetration and high path loss which can cause severe interference for FUEs within femtocells and create dead-zone area for them. Dead zone area is shown with red dashes in Figure 1. To overcome from the interference caused by MUE1, the FUEs within dead-zone area transmit with higher power. This cause severe interference at MBS and decreases received SINR from MUE1 and can create closed loop of positive interference, if interference is not mitigated properly.

To mitigate this problem, possible solution is to handover MUE to FAP when it enters into the house-hold premises. The handover can be made on the basis of different SINR thresholds and TTS as well as taking resources in accounts to prevent any disruptions for already allocated or reserved resources in macrocell/femtocells. This would allow MUE to use small power and remain connected to FAP to carry out its transmission. The advantage of this strategy is to reduce unnecessary handovers and maintain the performance of network and optimize the throughput, data rates and network's capacity. Consider

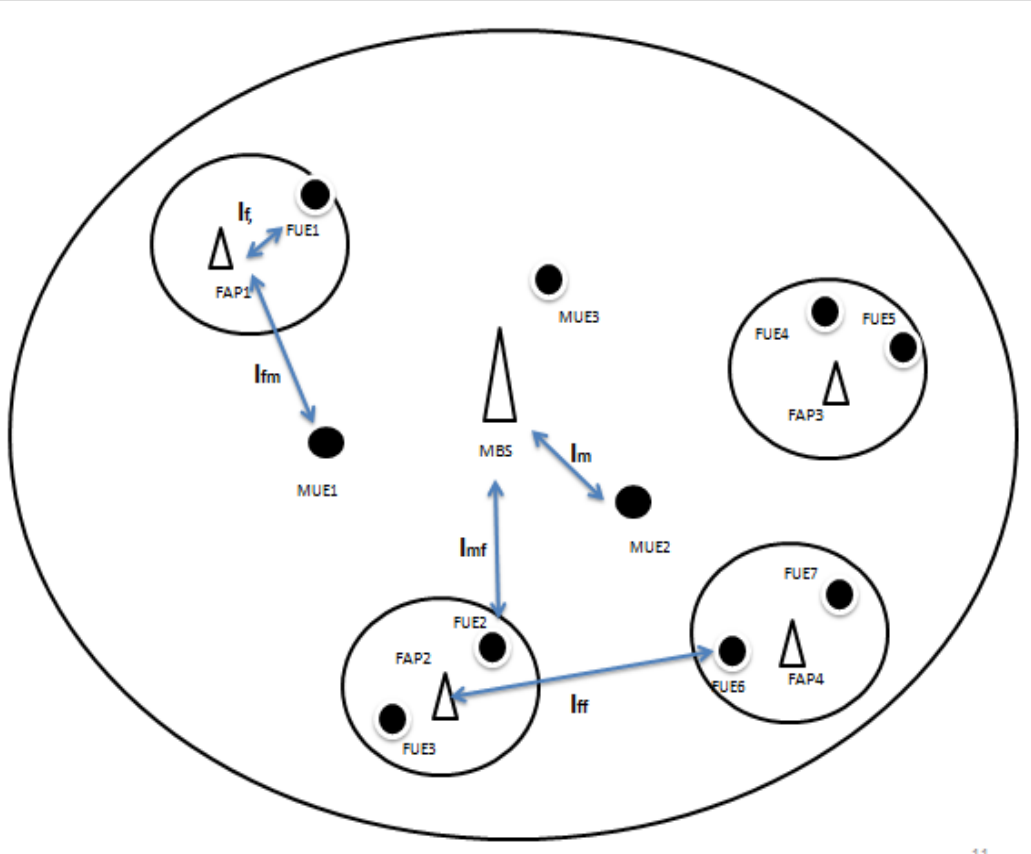

Figure 1: Co-channel interference in uplink transmission. 
set of parameters to prevent unnecessary ping pong affect as well as allocate resources to only those nodes that require urgent handover to maintain their services.

According to a study [20], interferences at FAP and MBS can be due to different factors which can degrade the performance of network. Interference caused by the MUEs using same channel and neighboring FUEs in other FAP can be the main reason for causing interference on FAP. Along with that, FUEs within the same Femtocells can also cause intra-cell interference while MUEs and FUEs using same channel within the coverage area of macrocells are responsible for interference at MBS. Interference from different entities can be calculated from the given formulas. In Figure 1, we can see interference between neighboring femtocells $I_{f f}$, interference between FUEs and femtocells $I_{f}$, interference between FAP and MUEs $I_{f} m$, interference between macrocell and MUEs $I_{m}$ and interference between MBS and FUEs $I_{m} f$. Interference calculations are made by assuming values of power received by MBS $P^{c}$, power received by FAP and $P^{f}{ }_{r}$ outdoor attenuation factor taken from previous study [24]

\section{The neighboring femtocell interference $I_{f f}$ at $\boldsymbol{F} \boldsymbol{A P} \boldsymbol{P}_{j}$}

The interference caused at FAP due to the FUEs in nearby femtocells can be calculated as [20]:

$$
I_{f, f}=P_{r}^{f} \frac{g c\left(X_{i}\right)}{g_{f}\left(R_{f}\right)}
$$

Where $X_{i}$ is the distance between one FAP to the other and Rf is radius of femtocells.

\section{The femtocell $F_{j}$ intra-cell interference $I_{f}$ at $F A P_{j}$}

The interference caused by FUEs on the serving FAP also degrades SINR at FAP and calculated as [20]:

$$
I_{f}=\left(U_{f}-1\right) P_{r}^{f}
$$

Where $U_{f}$ is the number of FUEs within the Femtocell while $P_{r}^{f}$ is the received power of FAP.

\section{The macrocell interference $I_{m f}(i)$ at $F A P_{j}$}

The interference on FAP from each MUE can be calculated as [20]:

$$
I_{m f}(i)=P_{r^{c}} \frac{g c\left(Y_{i}\right)}{g c\left(X_{i}\right)}
$$

The sum of all interferences from MUEs on FAP can be calculated from:

$$
I_{m f}=\sum_{i=1}^{N c} I_{f c}(i)
$$

Where $X_{i}$ is the distance between MUEs and MBS while $Y_{i}$ the distance between FAP and MUEs. $N_{c}$ is the number of MUEs in the macrocell.

\section{Macrocell interference at MBS}

MUEs using the same channel are responsible for causing interference at MBS. It can be calculated by assuming that $\mathrm{Nc}$ are the numbers of MUEs using same channel while $P_{r}^{c}$ is the power received at MBS.

$$
I_{m}=\left(N_{c} 1\right) P_{c}
$$

\section{Femtocell interference at MBS}

Femtocells within the coverage area of MBS have Uf number of
FUEs which can cause interferences on MBS. Assume that interference of FUEs within the same femtocell has almost same affect because distance of those FUEs is almost same from FAPs. Assuming that $X$ is the distance between $F A P_{i}$ and MBS, the interferences at MBS from each $(F U E)_{j}$ in femtocell Fi can be calculated:

$$
I_{f m}\left(F_{i, j}\right)=P_{r}^{f} \frac{g c\left(X_{i}\right)}{g f\left(R_{f}\right)}
$$

Therefore, total interference at MBS from all FUEs from the femtocells within its coverage can be given as:

$$
I_{f m}=\sum_{i=1}^{N_{f}} \sum_{j=1}^{U_{i}} I_{f m}\left(F_{i, j}\right)
$$

\section{Parameters}

From the previous studies, conclusion can be made that there is a need of system's design which should be optimized and good enough for handling interference problem between macrocells and femtocells by using efficient handover technique. Now consider three parameters for making decision of handover because most of the previous research has focus on these parameters independently but this research aim to show that by considering all equally important, improved solution can be achieved for reducing co-channel interference and unnecessary handover.

\section{Time to stay (TTS)}

Time to stay is defined as the predicted time of stay of mobile user wtihin coverage area of FAP. When node enters into the coverage area of FAP, it's time to stay depends on its velocity. We are using random waypoint as our mobility model.Let's consider that the distance covered by the node in two time span according to the predicted velocity of respective node. Calculate Time to stay by considering both distance and velocity by following formula [20].

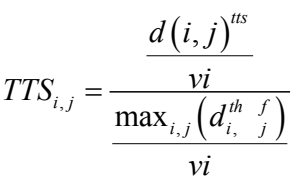

$$
\begin{aligned}
& d_{i, j}^{t s s}=\frac{R_{f}}{\operatorname{Sin} \theta_{i, j}} \cdot \sin \left(\theta_{i, k}+\arcsin \left(\frac{d_{i, j}}{R_{f}}\right) \cdot v_{i}\right)
\end{aligned}
$$

Where $d_{(i, j)}$ is the distance between FAP and MUE while $T T S_{(i, j)}$ is the time node has spent within the coverage of that FAP. (TTS)thF is the normalized TTS that is required for FAP to carry out handover if threshold exceeds required limit which means that if node stays longer then it's should be handover to FAP to avoid dead zone area and degradation of SINR at MBS. TTS depends on the distance and velocity of node. By using trajectory and velocity of node, $T T S_{i, j}$ can be calculated that can provide us the predicted stay of node. If the average value of $T T S_{i, j}$ is less than $(T T S)_{t h F}$, then it is likely to have few handovers because handover can only perform when $(T T S)_{t h F}$ become smaller than $T T S_{i, j}$. Because (TTS) $)_{t h F}$ is a threshold value of FAP to perform handover, if nodes till the time required for handover.

\section{Signal-to-interference-plus-noise ratio (SINR)}

SINR is defined as the quantity for channel capacity and it acts as the upper bound for the rate on which information is transmitted. It is power of signal of certain node divided by the sum of all interferer around that node and power of background noise. In wireless networks, it is used to check the quality of the connection. In telecommunication, 
UE is connected to the Base station with highest SINR for seamless connectivity and capacity. Previously RSS was used as the measure of handover decision by comparing the RSS of UE with predefined value. But it doesn't meet throughput because it fails to provide the data rates demanded by the UE for carrying out its services. Due to which SINR is proposed as the indicator of handover decision because it ensure to meet required data rates. In this paper, SINR is used instead of RSS as one of the parameter of handoff when MUE moves from MBS to FAP due to increase of distance between MUE and MBS, the received SINR at MBS decreases. Due to which MUE tries to transmit with high power. It also has adverse effect at FAP as MUE will create dead zone area for nearby FUEs already connected to that FAP. To overcome this issue, handover is being made by comparing different threshold of SINR to make decision.

In this research [20], two SINR thresholds are proposed: actual SINR $\gamma_{f H}$ and preliminary SINR thresh-old $\gamma_{f D H^{*}}$. Actual threshold is the SINR threshold on which handover should be made without any other consideration. However, in this research preliminary SINR is introduced threshold because when system will check right from the moment when performance of network starts degrading and then both by comparing the SINR threshold depending upon its predicted time within that coverage, precise decision needs to be taken that either it's important to handover to nearby FAP or to wait little longer before conditions go worst. The values are dependent on the QoS provided by femtocells to MUE. For example, for real time services threshold is set much higher than non real time services because they need high bandwidth for smooth service, interference level and traffic load. SINR at FAP and MBS can calculated on the basis of co-channel interference is given as [20]:

$$
\begin{aligned}
& (\operatorname{SINR})_{F A P}=\frac{\left(G_{f} \cdot P_{r}^{f}\right)}{\left.\left(I_{f f}+I_{f m}+I_{f}\right)+\sigma_{f}\right)} \\
& (\operatorname{SINR})_{M B S}=\frac{\left(G_{M} \cdot P_{r}^{c}\right)}{\left(I_{m f}+I_{m}+\sigma_{m}\right)}
\end{aligned}
$$

According to equation, all interference at FAP and MBS from MUEs and FUEs should be taken into account.

\section{Availability of resources}

Radio resource management is very crucial for maximizing the efficient utilization of resources. As bandwidth is limited, so there should be precise mechanism for handover decision is which UE should be handed over in the condition when resources are available in the cell. Any new request is only accepted if QoS conditions are met [25]. In this paper, resource allocation method is proposed which takes decision for handover after checking SINR and TTS threshold in a way that there is minimum interference between the adjacent nodes because both macrocells and femtocells are using same frequency band. The algorithm used by us for resource assignment is fractional frequency reuse-based graph connectivity (FFR-GC). By using this algorithm, MUEs located on the edges will be less suffered by the interference. FFR-GC is divided into two parts. Firstly, sub-bands are allocated to macrocells and femtocells. Secondly, by using graph theory the requested band will be assigned in a way that will reduce interference among the nearby femtocells [18].

Macrocell radio resource allocation: Technique of FFR-GC was proposed in a study [18], which is used in this research as a measure to identify either sub-band is available for handover and to make decision in a way that will help in optimizing the interference as much as possible. Lets we consider that we have 4 frequency sub-bands (A1, A2, A3, A4). Firstly, macrocell is divided into a centre zone $(\mathrm{C} 1, \mathrm{C} 2$, C3) and three edge zones (E1, E2, E3), as shown in Figure 2.

Femtocells radio resource allocation: In this phase, resource is allocated to femtocells is such a way that sub-band $A 1$, which is $2 / 3$ of total bandwidth, is further divided into 6 sub-bands and is allocated to the edges where it is used by femtocells. Along with this, sub-bands that are not allocated to macrocells in centre-zone and edges are used by femtocells to avoid interference between macrocell and femtocells as shown in Figure 3. But the interference between femtocells due to reuse of sub-bands is still a problem.

\section{Proposed algorithm for resource allocation}

In this case, graph theory is used in which with the help of undirected colored graph the femtocells can be identified about the bands they are using, so that interference can be avoided by not allocating same channels to immediate neighboring femtocells. Maximal Independent Set (MIS) is proposed for finding the independent set and then by the help of those set resources can be allocated to femtocells for avoiding interference. MIS is a good algorithm for reduction of interference between the femtocells using same sub-bands but cannot use same channel [18]. For each simulation, how many sub-bands are used by FUEs within femtocells are recorded by maintaining matrix and check which sub-band can be allocated according to geographical condition of femtocell in cellular environment to avoid any type of interference between MBS as well as neighboring femtocells.

An algorithm is proposed that aims to reduce interference among macrocells and femtocells by considering the threshold of SINR and TTS [20] along with the availability of resources to take decision of handover [18]. The focus of previous research was to assign bands to

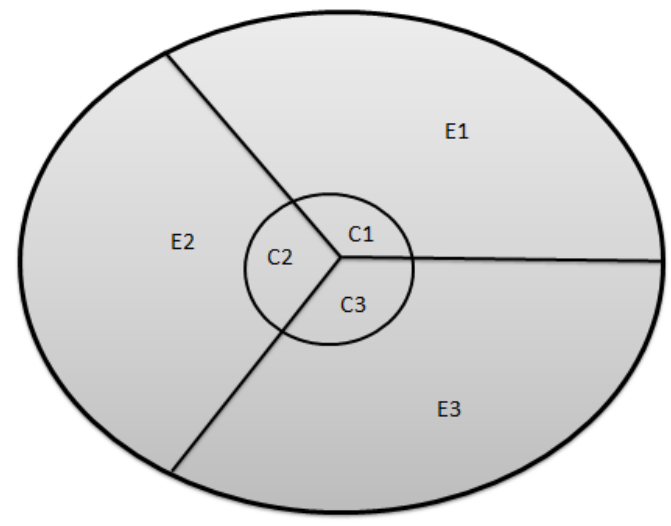

Figure 2: Area division w.r.t centre and edges.

\begin{tabular}{|c|c|c|c|}
\hline Area & Cells & Macrocell & Femtocell \\
\hline \multirow{4}{*}{ Centre } & C1 & A1 & A3, A4 \\
\cline { 2 - 4 } & C2 & A1 & A2, A4 \\
\cline { 2 - 4 } & C3 & A1 & A2, A3 \\
\hline \multirow{4}{*}{ Edges } & E1 & A2 & A1, A3, A4 \\
\cline { 2 - 4 } & E2 & A3 & A1, A2, A4 \\
\cline { 2 - 4 } & E3 & A4 & A1, A2, A3 \\
\hline
\end{tabular}

Figure 3: Distribution of sub-bands. 
neighboring femtocell in a manner to avoid intereference and handover was not considered in that paper. The main advantages of proposed algorithm are that it is considering all important parameters altogether. The movement of MUEs will be based on random way point (RWP) for predicting the future movement of MUEs that would be effective for taking decision about the time MUEs could stay within the coverage of femtocells. If TTS is greater than the required threshold time for FAP, then it will check for SINR and resources at FAP and further decision for handover will be based on their values.

In Figure 4 undirected graph in shown in which vertices's are representing femtocells while edges are representing interference between femtocells. The undirected graph is formed on the basis of assumption i-e let's say distance threshold is 40 meters. If distance between neighboring femtocells is less than or equal to 40 meters,then there will be interference between femtocells according to assumption. Adjacent matrix is formed by checking which band can be used by femtocell according to geographical location i-e by taking edges and center band assignment as given in Figure 5 .

\section{Maximal independent set (MIS)}

An independent set, in graph theory, is a set with no neighboring vertices [26]. Let assume that $G(V, E)$ is an undirected graph in which

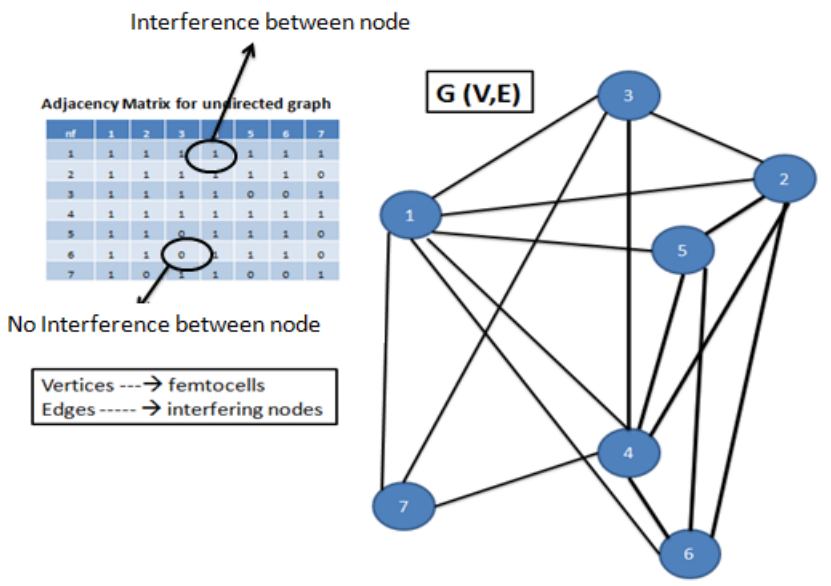

Figure 4: Undirected graph $\mathrm{G}(\mathrm{V}, \mathrm{E})$ and respective adjacency matrix.
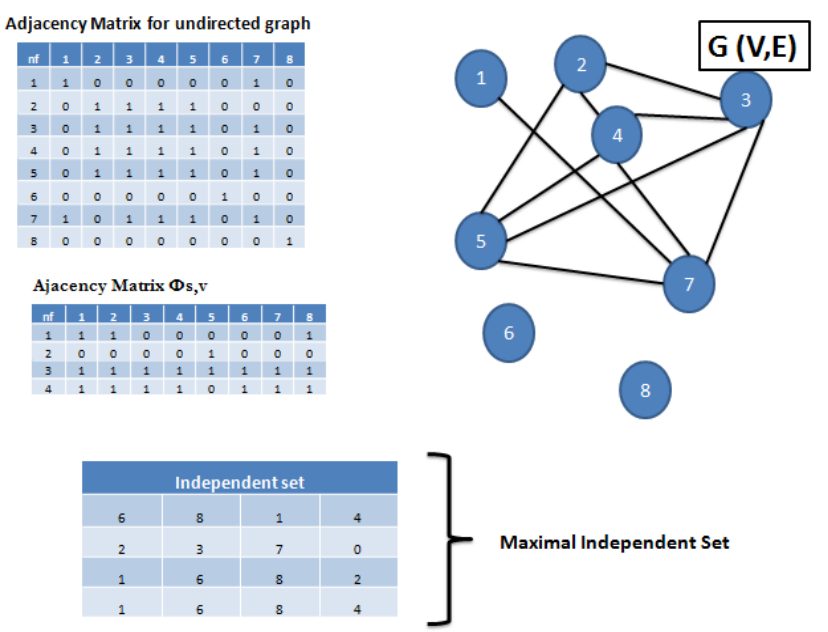

Figure 5: Maximal independent set from proposed scheme.
$\mathrm{V}$ is representing vertices while $\mathrm{E}$ is representing the edges. In this case, there is need to find set $\mathrm{K}$ in which no two adjacent vertices should be connected by an edge. The size of an independent set depends upon the the number of vertices it has. Independent sets are also called internally stable sets. In graph theory, finding maximal clique or maximal independent set is the basic problem and many algorithms are proposed for optimal solution. Algorithm used for listing all maximal independent sets can be used for solving many NP graph problems.

In the resource allocation method [18], as shown in Figure 4, undirected graph $\mathrm{G}(\mathrm{V}, \mathrm{E})$ is formed first, where $\mathrm{V}$ is femtocells and $\mathrm{E}$ is showing interference between femtocells. This graph is formed on the basis of distance between the Femtocell. Assumption is made that if distance is two times the radius of femtocells, then interference is happening between femtocells. Which band can be used by femtocells can be found by $\Phi_{s, v^{\circ}}$ With reference to $\Phi_{s, v^{*}}$, sets of femtocells will be extracged that are independent to each other and can use same bands by using MIS algorithm. Following are the steps of MIS algorithm that are discussed in a study [18] that followed in this research for resource allocation. Lets assume that we are assigning 4 bands to 6 femtocells. Now we should assigned them in a manner that will not cause any interference as shown in Figure 5:

Following steps should be followed to assign bands and there results are shown in Figure 5.

- Convert the results into undirected graph $g=(v, e)$ where $v$ denotes vertices while e denotes edges of graph.

- Then find a graph Gm (vm, em) with each sub-band which tells the information of femtocells to which sub-bands can be assigned.

- Femto gateway (FGW) will manage this information because all information regarding location, neighbors etc are managed by FGW

- In this step a set $\mathrm{k}$ is find out on the basis of MIS which include all femtocells to which sub-bands can be assigned without interference.

\section{Criteria proposed for handover}

Let consider that MUE starts moving towards femtocells and away from MBS. As the distance between MUE and MBS increases, SINR decrease. When MUE moves into the premises of femtocells especially within the indoor environment, it needs to transmit with high power due to wall's penetration and high path loss. When MUE starts transmitting with high power, it can create dead-zone area for near-by FUEs. Therefore, there is a need to initiate handover process and check the following conditions before it get worst as shown in Figure 6 .

Case 1: If MUE is moving toward femtocells and SINR starts degrading. If the SINR at FAP $(S I N R)_{F A P}$ is greater than preliminary SINR $\gamma_{f H}$ as well as TTS of MUE TT $S_{i, j}$ in femtocells is greater than normalize TTS threshold $T T S_{t h F}$, then MUE is eligible for handover. Otherwise, if SINR at FAP $(\text { SIN R })_{F A P}$ is greater than preliminary SINR $\gamma_{f} p H$ but TTS of MUE $T T S_{i, j}$ in femtocells is lower than normalized TTS threshold $T T S_{t h F}$, then there will be no handover.

- If $(S I N R)_{F A P}>\gamma_{f p H}$ and $T T S_{i, j}>T T S_{t h F}$, then handover takes Place

- If $(S I N R)_{F A P}>\gamma_{f p H}$ and $T T S_{i, j}<T T S_{t h F}$, then no handover takes place

Case 2: If the SINR at FAP $(\operatorname{SIN} R)_{F A P}$ becomes less than preliminary 


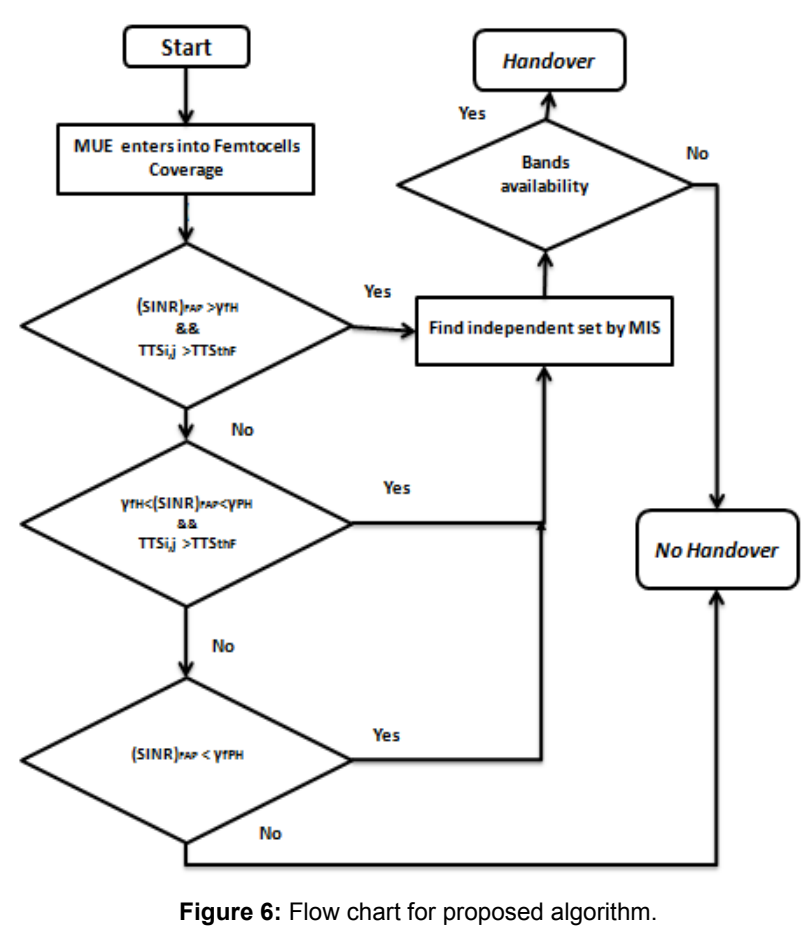

SINR $\gamma_{f} H$ but greater than actual SINR at FAP. Then check TTS of MUE $T T S_{i, j}$ within femtocells. If TTS of MUE $T T S_{i, j}$ in femtocells is also greater than normalized TTS threshold $T T S_{t h F}$, then MUE will be eligible for handover. If TTS of MUE $T T S_{i, j}$ in femtocells is less than normalized TTS threshold $T T S_{t h F}$, then no handover takes place.

- $\gamma_{f p H}<(S I N R)_{F A P}<\gamma_{f H}$ and $T T S_{i, j}>(T T S)_{t h F}$, then handover occurs

- $\gamma_{f p H}<(S I N R)_{F A P}<\gamma_{f H}$ and TT $S_{i, j}<(T T S)_{t h F}$, no handover occurs

Case 3: If MUE keeps on moving toward femtocells then SINR will degrade at FAP $(\operatorname{SINR})_{F A P}$ and if it becomes less than actual SINR $\gamma_{f H}$ , then handover will take place without further checking either TTS of MUE $T T S_{i, j}$ is less or greater than normalized threshold $(T T S)_{t h F^{*}}$ There will be no further checking for resource as well.

- If $\gamma_{f H}<(S I N R)_{F A P}$, handover will take place. This case is not independent on TTS.

\section{Check for resource availability}

After checking the all cases for SINR and TTS, check for preliminary conditions. If they satisfy the cases required for the handover except in Case 3, then further checking will be done for resources either available in femtocells for assigning to the MUEs that want to associate with FAP. For this, first identify the independent set by using MIS method. FGW has the information about the location, sub-bands availability in femtocells. According to the frequency allocation technique defined in a study [18], sub-bands allocated to femtocells cannot be used by macrocell. The resources are allocated among the femtocells in order that interference will be minimal and no two adjacent femtocells can use same sub-bands. This means that handover can be made only be resources are available in femtocells, otherwise there will be no handover.

The resource available decision is made by comparing the condition that if handover is necessary w.r.t SINR and time to stay but required resources are not available for FAP, according to MIS to avoid interference, on which handover is required then no handover will be performed and vice versa our proposed algorithm is shown in Figure 6 . The handover decision will optimize handover because only those handover will perform that are necessary.

\section{Performance Analysis of Network}

In this chapter, there will be discussion about the performance analysis of network by over viewing the outage probability, user sum rate and capacity by using proposed strategy in chapter 3 .

\section{Outage probability at FAP}

The connection between FUE and FAP will be outage if SINR received at FAP becomes less than minimum SINR threshold i.e $(\text { SINR) })_{\mathrm{FAP}} \geq \gamma$ fo Therefore, there should be more than one FUE $U_{f}$ in femtocell, so they can transmit with minimum power for transmission. The outage probability at FAP can be calculated as:

$$
\begin{aligned}
& P_{\text {out }}^{f}=P\left(\frac{\left(G f \cdot P \gamma^{f}\right)}{\left(I(f, f)+I(f, c)+I(f, \text { in })+\sigma_{f}\right)} \leq \gamma_{f o} \mid U_{f} \geq 1\right) \\
& \left\{\begin{array}{ll}
0, & (\operatorname{SINR})_{F A P} \geq \gamma f_{o} \\
\gamma f_{0}-\frac{\left(G_{f} \cdot P_{\gamma}^{f}\right)}{\left(I_{f, f}+I_{f, c}+I_{f, \text { in }}+\sigma_{f}\right)} & (\operatorname{SINR})_{F A P}<\gamma f_{o}
\end{array}\right\}
\end{aligned}
$$

This subject to: $P^{\text {out }}\left(N_{f}, N_{c}\right) \leq \varepsilon_{f}$ Where $\varepsilon_{f}$ denotes the requirement of outage probability at FAP while $\sigma_{f}$ is the log normal attenuation for FAP.

\section{User sum rate}

The user sum rate of all users using same channel $\mathrm{n}$ can be calculated as: 


$$
R_{M F}^{n}=\theta \sum_{i=1}^{N_{f}} \log _{2}\left[1+\sin _{M_{i}}^{c(n)} P_{r}^{c}\right]+(1-\theta) \sum_{i=1}^{N_{f}} \sum_{j=1}^{U_{i}} \log _{2}\left[1+\sin _{F k j}^{(n)} P_{r}^{f}\right]
$$

In this equation, $\log _{2}\left[1+\sin _{M_{i}}^{c(n)} \cdot \mathbf{P}_{r}^{c}\right]$ is the achievable rates of MUEi in the macrocell while $\log _{2}\left[1+\sin _{F k j}^{(n)} \cdot P \gamma^{f}\right]$ is the achievable rates of FUE $_{\mathrm{kj}}$ in each femtocells as explained in a study [26].

\section{Numerical Results}

This research shows the proposed schemes in terms of outage probability and SINR with existing techniques. Table 1 is showing the parameters that we are assuming in our research for getting simulation results. The number of femtocells are varying from 1 to 100 in one macrocell coverage to find out the influence of this variation. The MUEs and FUEs are randomly distributed in the network and random way-point mobility model is used for MUEs movement for each time slot. To show the benefits, proposed algorithm will be compared with the results that are achieved in handover with the algorithms that are only considering handover on the basis of SINR and TTS [20]. We have assumed ten MUEs in the coverage area of macrocells, while five FUEs within each femtocell.

In the Figure 7, TS is showing the number of handover on the basis of TTS and SINR, while TSR is showing number of handovers on the basis TTS, SINR and resources available. We can see that unnecessary handovers are increasing exponentially with increase in number of femtocell while we can achieve significant reduction in handovers by using our proposed algorithm. In Figure 8, it is shown that SINR is decreasing with the increase in femtocells rapidly, if we compare it with previous scheme [20]. But we can see that SINR is getting slightly

\begin{tabular}{|c|c|c|c|}
\hline Parameters & Value & Parameters & Value \\
\hline$R_{c}$ & $500 \mathrm{~m}$ & $R_{f}$ & $20 \mathrm{~m}$ \\
\hline$P_{r}^{f}$ & $10 \mathrm{~mW}$ & $P_{r}^{c}$ & $1 \mathrm{~mW}$ \\
\hline$t_{m}$ & 50 & $t_{f}$ & 10 \\
\hline$d o c$ & $100 \mathrm{~m}$ & $d o f$ & $5 \mathrm{~m}$ \\
\hline$\gamma f H$ & 3 & $\gamma f P H$ & 5 \\
\hline$T T S_{\text {thF }}$ & $8 \mathrm{~min}$ & $U_{f}$ & 5 \\
\hline$N_{c}$ & 10 & $v$ & $20 \mathrm{~km} / \mathrm{hr}$ \\
\hline$a$ & 4 & $\beta$ & 2 \\
\hline$d t h r e s h o l d$ & $40 \mathrm{~m}$ & $f$ & $2 \mathrm{GHz}$ \\
\hline
\end{tabular}

Table 1: Differences between different cellular architecture.

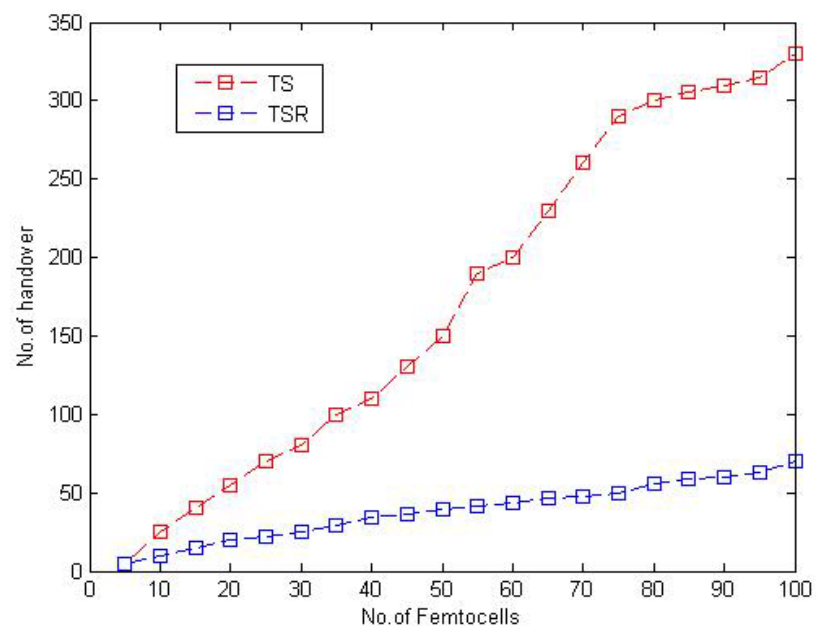

Figure 7: Number of handovers in femtocells. better with our proposed scheme. This is because we are considering resources and we are not performing handover if resources are not available. While SINR received from method proposed in a study [20] is degrading more rapidly because they are assuming infinite resources and SINR is regarding due to unnecessary handover. In Figure 9, we can see that we are getting better results for user sum rate from our proposed scheme and it decay slowly than the previous scheme proposed [20].

In Figure 10, femtocell outage probability calculated from our proposed scheme is increasing less rapidly then it was with previous scheme [20]. Because we are considering resources before handover, connection between FAP and FUE is an outage if resources are not available. Resources need to be taken into account while performing handover. The results of SINR received at FAP and OP on the basis of TS scheme are better than those from TSR scheme, because it is assumed in the study that resources are available for handover. While the results from previous techniques [20] shows that handover decision is made without considertaion of resources is showing its effects on SINR, OP and User Sum Rate. However, in the proposed scheme, since there are limited resources, therefore SINR falls below when resources are not available and it starts decreasing with increase in number of

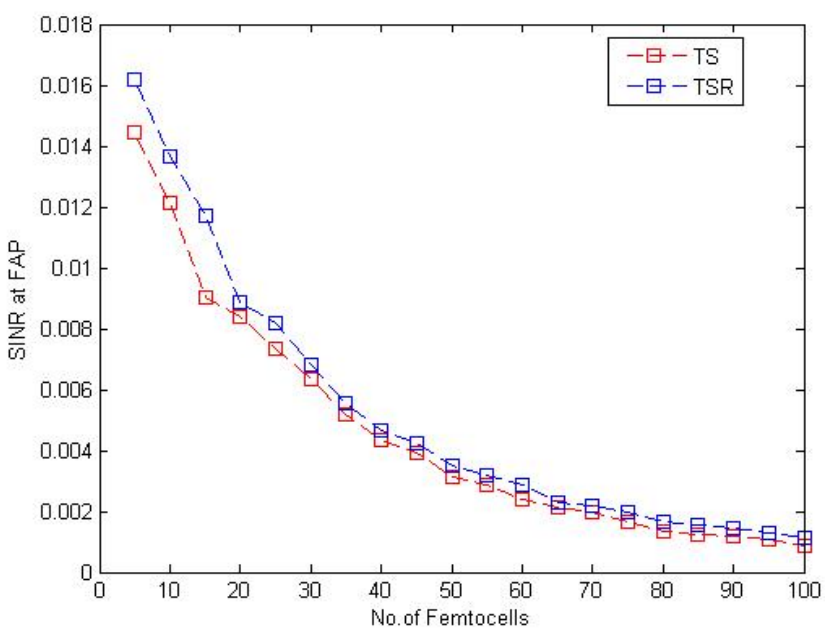

Figure 8: SINR received at FAP.

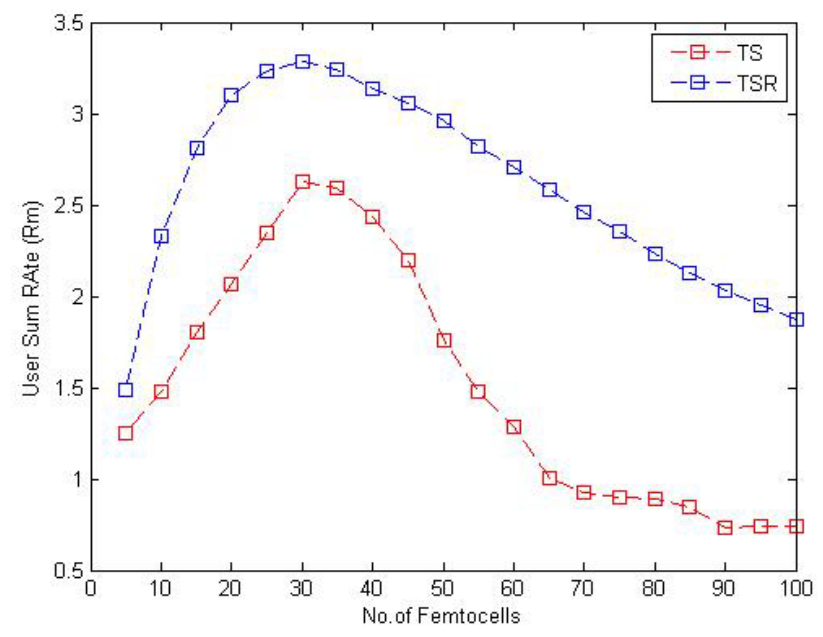

Figure 9: User sum rate. 


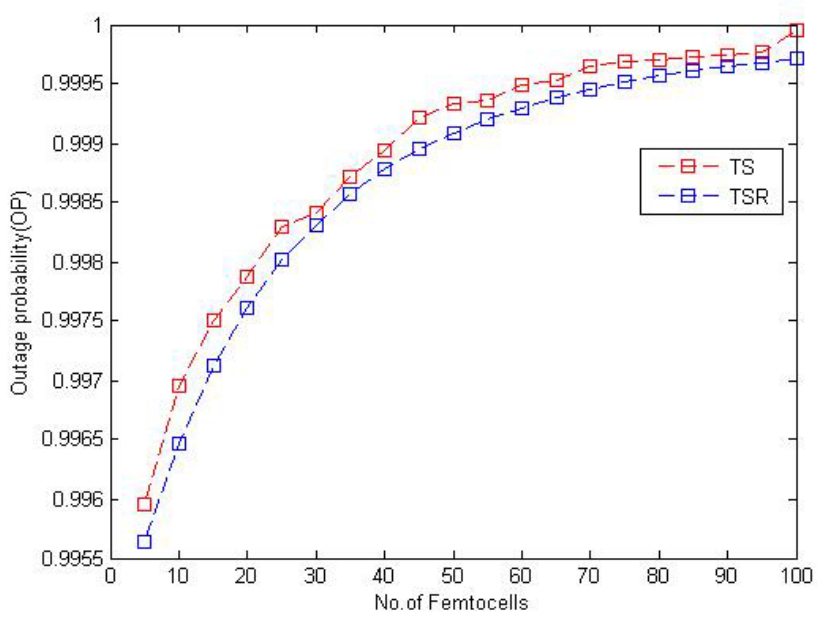

Figure 10: Femtocell outage probability.

femtocells. From the following results, we can say that by maintaining SINR and OP as shown in a study [20], we can improve sum rate from our proposed scheme as well as reduce number of handovers.

\section{Conclusion and Future Work}

In this research, interference and resource allocation strategy is used for making handover decision to avoid unnecessary handovers. From the results, it can be concluded that promising results can be achieved if handover is made on the basis of SINR, TTS and resource availability. By comparing the proposed method with the previous research [20], which two important parameters i-e SINR and TTS are considered, conclusion can be made that there is a decrease in handover, if resource availability is also considered. From previous studies, this can be concluded that SINR, TTS and resources are three main parameters that need to be checked. In future, there is need to dig down about more important parameters that can be checked for improving results. In this research, there is limitation because GPS data is not used in future results can be improved by taking realistic approach. Secondly RWP mobility model is used in this research, other mobility models can be used according to particular scenario. Number of FUEs and MUEs can be increased in future research to check impact of proposed algorithm in populated area. In this research, femtocell are distributed in unplanned manner, in future if requirement is for planned femtocell architecture, then this proposed algorithm can be used according to the requirement.

\section{References}

1. Cheng-Xiang W, Fourat H, Xiqi G, Xiao-Hu Y, Yang Y, et al. (2014) Cellular architecture and key technologies for $5 \mathrm{~g}$ wireless communication networks. IEEE Communications Magazine 52: 122-130.

2. Adnan A, Hamid A, Mojdeh A (2013) A survey on mobile data offloading: technical and business perspectives. IEEE Wireless Communications 20: 104112

3. Cisco Visual Networking Index (2013) Global mobile data traffic forecast update 2012-2017.

4. John H, Payam M (2012) Enabling small cell deployment with hetnet. IEEE Wireless Communications 19: 4-5.

5. Ludovic Fournier. Lte (2011).
6. Ronny YK, Jin SK, Etemad K (2009) Wimax femtocell: requirements, challenges, and solutions. IEEE Communications Magazine 47.

7. Xavier G, Peter L, Christer Q (2013) Small cell densification requirements in high capacity future cellular networks. In Communications Workshops (ICC) 2013 IEEE International Conference, pp: 1112-1116.

8. Holger C, Lester TH, Louis GS (2008) An overview of the femtocell concept Bell Labs Technical Journal 13: 221-245.

9. Amitabha G, Nitin M, Rapeepat R, Bishwarup M, Mark C, et al. (2012) Heterogeneous cellular networks: From theory to practice. IEEE Communications Magazine 50.

10. Byungjin J, Seungjae S, Ingook J, Nak WS, Yoon H (2011) A smart handove decision algorithm using location prediction for hierarchical macro/femto-cel networks. In Vehicular Technology Conference (VTC Fall) (2011) IEEE, pp: $1-5$.

11. Thomas J, Irina B, John T, Ingrid M, Kurner T (2010) Handover paramete optimization in Ite self-organizing networks. In Vehicular Technology Conference Fall (VTC 2010-Fall), 2010 IEEE 72nd, pp: 1-5.

12. Shih-Jung W (2011) A new handover strategy between femtocell and macrocell for Ite-based network. In Ubi-Media Computing (U-Media), 2011 4th International Conference on IEEE, pp: 203-208.

13. Md Rejwan H, Azm Ariful HR, Rahman A (2015) Handover management in heteroge-neous network. In Computer and Information Technology (ICCIT) 2015 18th International Conference on IEEE, pp: 87-92.

14. Er Limbad S, Mohit B (2015) A survey of handover mechanism with mobility management in femtocell \& macrocell for LTE cell.

15. Carlos HM de L, Kaveh G, Mehdi B, Allen BM, Latva-aho M (2010) A stochastic association mechanism for macro-to-femtocell handover. In Signals, systems and computers (ASILOMAR), 2010 conference record of the forty fourth ASILOMAR conference on IEEE, pp: 1570-1574

16. Jae-Wook L, Sang-Jo Y (2016) Probabilistic path and data capacity based handover decision for hierarchical macro-and femtocell networks. Mobile Information Systems, 2016.

17. Sungkwan Y, Jai-Jin J, Youngwoong K, Eui-Jik K (2015) Resource efficient handover strategy for Ite femtocells. International Journal of Distributed Sensor Networks 11: 962-837.

18. Gwo-Jong Y, Khac HN (2016) A novel downlink interference management mechanism for two-tier ofdma femtocell networks. Journal of Advances in Computer Networks 4: 80-85

19. Dae KS, Wan C, Takki YU (2013) Statistically controlled opportunistic resource block sharing for femto cell networks. Journal of Communications and Networks 15: 469-475.

20. Geng C, Jun Z, Lianfeng S (2016) A preset threshold based cross-tier handove algorithm for uplink co-channel interference mitigation in two-tier femtocell networks. Wireless Networks 22: 1819-1835.

21. Azita Laily Y, Siti Sabariah S, Norsuzila Y, Mohd TA (2013) Performance analysis of handover strategy in femtocell network. Journal of Communications 8: 724-729.

22. Mohmmad A, Francesco DC, Preben EM, Claudio R, Klaus I P (2007) Performance evaluation of received signal strength based hard handover for utran Ite. In Vehicular Technology Conference 2007. VTC2007-Spring. IEEE 65th, pp: 1046-1050

23. Damar W, Peerapong U (2016) Optimum threshold for velocity consideredsinr based vertical handoff decision in hetnet. ECTI Transactions on Electrical Engineering, Electronics, and Communications 14: 65-72.

24. Vikram C, Jeffrey GA, Alan G (2008) Femtocell networks: a survey. IEEE Communications magazine 46

25. David LP, Akos LA, Alpa J, Jie Z (2009) Ofdma femtocells: A self-organizing approach for frequency assignment. In Personal, Indoor and Mobile Radio Communications, 2009 IEEE 20th International Symposium on IEEE, pp: 2202 2207.

26. Reuven B-Y, Keren C-H, Mohsen G, Gregory S (2017) Distributed approximation of maximum independent set and maximum matching. 